\title{
Oleksandr Stasiuk
}

Nationale Taras-Schewtschenko-Universität Kyjiw, Kyjiw

DOI: $10.19195 / 0435-5865.141 .22$

\section{Metaintertextuelle Markierungen des massenmedialen Interdiskurses in parlamentarischen Texten Deutschlands und Schwedens}

\section{Einleitung}

$\mathrm{Zu}$ den wichtigsten Fragen der gegenwärtigen Linguistik gehören die Vernetzung und das Zusammenwirken von Texten und Diskursen, die durch die Begriffe „Intertextualität“" und „Interdiskursivität" erfasst werden. Sie verbinden Texte und Diskurse miteinander, dienen als Mittel der Dialogizität und Verständigung zwischen den Menschen, tragen zu der Entwicklung von Textsorten und Diskursen bei.

Der Begriff „Intertextualität“" wurde von Kristeva eingeführt, die grundlegenden Ideen dazu wurden aber von Bachtin entwickelt (vgl. Kristeva (1980); Bakhtin (1975). Bis heute hat sich der Begriff ,Intertextualität“ in mehreren Wissenschaftsbereichen, u.a. in Textlinguistik, etabliert, und, wie Heinemann (1997: 21) bemerkt, ist ,zu einer Art Modebegriff in verschiedenen Wissenschaftsdisziplinen geworden“. Im weiteren Sinne wird unter der Intertextualität verstanden, dass „Texte miteinander interagieren, dass sie miteinander in Beziehung stehen“ (Heinemann 1997: 22).

Der Begriff „Interdiskursivität” ist relativ neu. Er wurde zum ersten Mal im Jahre 1992 von Fairclough (1992) gebraucht. Danach wurde die Interdiskursivität in Forschungsarbeiten meistens im engen Zusammenhang mit der Intertextualität behandelt. Die Interdiskursivität bezeichnet „die Vermischung von unterschiedlichen Gattungen, Diskursen und Stilen, die mit institutionellen oder sozialen Bedeutungen assoziiert sind, in einem einzelnen Text" (vgl. Wu 2011: 98-104).

Die Interdiskursivität ist vielen unterschiedlichen Bereichen der menschlichen Kommunikation eigen. Die politische Kommunikation zeichnet sich durch 
zahlreiche Einflüsse von verschiedenen Diskursen aus, was zur Erhöhung des Überzeugungspotenzials des Diskurses beiträgt (Schewtschenko 2009: 57). In der Parlamentskommunikation werden zahlreiche intertextuelle Fragmente gebraucht, die unterschiedlichen Textsorten und Diskurstypen entnommen sind. In den parlamentarischen Texten sind Elemente von dem politischen, juristischen, massenmedialen, wissenschaftlichen, literarischen Diskurs, Alltags-, Sportdiskurs zu finden. Der vorliegende Beitrag ist dem massenmedialen Interdiskurs in den parlamentarischen Texten Deutschlands und Schwedens gewidmet.

Der massenmediale Diskurs spielt eine wichtige Rolle in der Parlamentskommunikation Deutschlands und Schwedens. Dem Einfluss der Massenmedien auf den Verlauf der politischen Kommunikation wurden zahlreiche wissenschaftliche Publikationen gewidmet s. z.B. Jarren (2005) in Deutschland; Strömbäck (2009) in Schweden). Der Zusammenhang zwischen dem massenmedialen und dem parlamentarischen Diskurs erweist sich einerseits dadurch, dass die Arbeit des Parlamentes in den Massenmedien beleuchtet wird. An der Grenze des politischen und des massenmedialen Diskurses entstehen zahlreiche Texte, wie z.B. Texte, die in den parlamentarischen Zeitungen veröffentlicht werden (,Blickpunkt Bundestag” und „Das Parlament” in Deutschland, „Riksdag och departement” in Schweden). Andererseits lassen sich die Parlamentarier durch die Massenmedien über die aktuelle politische, wirtschaftliche, soziale Lage informieren, was zum Zitieren der Massenmedien in den parlamentarischen Texten führt.

Besonders groß ist die Rolle der Massenmedien im Schaufensterparlament, das von Burkhardt (2003: 6) folgenderweise definiert wurde:

[...] Schaufensterparlament (Deutscher Bundestag), das aus freien, gleichen und geheimen Wahlen hervorgeht, in dessen Plenum politische Entscheidungen in die Öffentlichkeit in kontroverser Debatte begründet und die eigentlichen Entscheidungsrituale (Abstimmungen) auch tatsächlich durchgeführt werden, in dem aber kaum mehr eine eigentliche Diskussion mit dem Ziel wechselseitigen Überzeugens stattfindet [...].

Das Ziel der Kommunikation im Parlament ist also, nicht die anderen Abgeordneten, sondern die Bürger zu überzeugen. Die Parlamentarier sprechen „zum Fenster hinaus“, wie es von Burkhardt (2003: 279) beschrieben wurde. In dieser Hinsicht spricht man von der „Doppeladressierung parlamentarischer Plenarkommunikation". ${ }^{1}$ Dabei kann die Intertextualität als Mittel der Überzeugung betrachtet werden, worauf z.B. Oliveira (2004: 10) hinweist. Aus diesen Gründen scheint es besonders wichtig zu sein, die interdiskursiven Einflüsse des massenmedialen Diskurses auf die gegenwärtige parlamentarische Kommunikation zu erklären.

Um zu bestimmen, ob ein Zitat zu dem massenmedialen Interdiskurs gehört, wurden die Textfragmente analysiert, die den Intertext beschreiben und in diesem Beitrag als Metaintertext bezeichnet werden. Unter dem Begriff „Metaintertext“

${ }^{1}$ Vgl. Burkhardt (1995: 78) 
verstehen wir die Textfragmente, die auf den Gebrauch des Intertextes von dem Redner hinweisen vgl. Gavenko (2011: 38-39). Nähere Betrachtung des Metaintertextes ermöglicht es zu bestimmen, zu welchem Diskurstyp das intertextuelle Fragment gehört. Betrachten wir folgendes Beispiel:

(1) Sven Morlok (Staatsminister für Wirtschaft, Arbeit und Verkehr in Sachsen): Ich möchte Sie einmal daran erinnern, wie einige Mitglieder Ihrer Koalition dieses Thema sehen. Vizekanzler Sigmar Gabriel sagte dazu am 7. Dezember der Braunschweiger Zeitung - ich zitiere -: "Die Finanzierung der Mütterrente erfolgt früher oder später aus Steuern, zumindest teilweise. Auf Dauer kann man das so nicht durchhalten, sonst steigen die Beiträge der Rentenversicherung." So der Vizekanzler. (DB: 95)

Hier finden wir neben dem Zitat auch seine Beschreibung. Der Autor des Textes weist auf den Autor des Intertextes und seine soziale Stellung hin (Vizekanzler Sigmar Gabriel), aber auch darauf, dass seine Worte in der „Braunschweiger Zeitung“" veröffentlicht wurden. Das letzte ist ein Hinweis auf die Zugehörigkeit des Zitats zum massenmedialen Diskurs. Solche Zitate wurden für die weitere Untersuchung ausgewählt.

Das Ziel der vorliegenden Untersuchung besteht darin, den Gebrauch der metaintertextuellen Markierungen des massenmedialen Interdiskurses in den parlamentarischen Texten Deutschlands und Schwedens zu charakterisieren und ihre Rolle für die Erforschung der Interdiskursivität aufzuzeigen.

Das Belegmaterial umfasst 154 Fragmente des Intertextes und Metaintertextes aus den parlamentarischen Texten Deutschlands und 179 Fragmente aus den parlamentarischen Texten Schwedens, die die metaintertextuellen Markierungen des massenmedialen Interdiskurses enthalten. Den stenographischen Berichten der Plenarsitzungen des Deutschen Bundestages und des Schwedischen Reichstages mit dem gesamten Textumfang von über 8000 Seiten wurden kontinuierlich Zitate entnommen, die dort explizit durch Anführungszeichen oder Texteinzug markiert sind. Die stenographischen Berichte enthalten eine Vielzahl von Textsorten, die den Kern des parlamentarischen Diskurses bilden: Abgeordnetenreden, Fragen der Abgeordneten an die Minister, Regierungserklärungen, Zwischenrufe und Zwischenfragen u. a. m., und sind repräsentativ für die Parlamentskommunikation.

\section{Lexikalisch-semantische Charakteristiken der metaintertextuellen Markierungen des massenmedialen Interdiskurses in parlamentarischen Texten Deutschlands und Schwedens}

Um den massenmedialen Interdiskurs aus den parlamentarischen Texten auszusondern, wurde es im Metaintertext nach den Hinweisen auf die Zugehörigkeit 
des Intertextes zum massenmedialen Diskurs gesucht. Sie gehören zu dem semantischen Bestand der Lexeme des Metaintertextes. Der Metaintertext enthält Lexeme mit folgenden Hinweisen, die als semantische Merkmale realisiert sind:

1. Der Name der Zeitung, der Zeitschrift, des Fernseh- oder Rundfunksenders, die als Quelle des Intertextes dienen: z.B. ARD, Bild, Focus, Frankfurter Allgemeine Zeitung, Spiegel, Welt; Aftonbladet, Dagens Industri, Svenska Dagbladet, Sveriges Radio, SVT.

2. Die Textsorte des Intertextes: z.B. Beitrag, Bericht, Interview, Kommentar, Presseerklärung, Pressemitteilung; debattartikel, intervju, ledare, pressmeddelande, reportage.

Dabei ist es zu berücksichtigen, dass einige Textsorten zu mehreren Diskurstypen gehören. Man unterscheidet z. B. Zeitungs- und Forschungsartikel. Deshalb muss es im Falle des allgemeinen Hinweises Artikel überprüft werden, ob das intertextuelle Textfragment den Massenmedien entnommen wurde. Dabei können sowohl weitere metaintertextuelle Markierungen, als auch der Intertext selbst behilflich sein.

3. Der Medientyp der Quelle, aus der zitiert wird: Magazin, Nachrichtenportal, Presse, Zeitschrift, Zeitung; lokaltidning, tidning, tidskrift.

4. Die Soziale Rolle des Autors des Intertextes, z. B. sein Beruf oder Anstellung: Journalist, Kulturkorrespondentin; chefredaktör, journalist, kronikör.

5. Der Hinweis darauf, dass der Intertext den Medien entnommen ist: Medien; medier, massmedier.

Insgesamt enthalten die analysierten Belege 227 lexikalisch-semantische Hinweise auf ihre Zugehörigkeit zu dem massenmedialen Diskurs in den parlamentarischen Texten Deutschlands und 303 Hinweise in den parlamentarischen Texten Schwedens. Ihre Verteilung nach den oben genannten Gruppen ist in der Tabelle 1 dargestellt.

Tab. 1 Lexikalisch-semantische Gruppen der metaintertextuellen Hinweise

\begin{tabular}{|c|c|c|c|c|c|c|c|}
\hline \multirow[t]{2}{*}{ Nr. } & \multirow{2}{*}{$\begin{array}{l}\text { Lexikalisch-semantische Gruppe der } \\
\text { intertextuellen Markierungen }\end{array}$} & \multicolumn{2}{|c|}{$\begin{array}{l}\text { Parlamenta- } \\
\text { rische Texte } \\
\text { Deutschlands }\end{array}$} & \multicolumn{2}{|c|}{$\begin{array}{l}\text { Parlamenta- } \\
\text { rische Texte } \\
\text { Schwedens }\end{array}$} & \multicolumn{2}{|c|}{ Insgesamt } \\
\hline & & $\begin{array}{l}\text { An- } \\
\text { zahl }\end{array}$ & $\%$ & $\begin{array}{l}\text { An- } \\
\text { zahl }\end{array}$ & $\%$ & $\begin{array}{l}\text { An- } \\
\text { zahl }\end{array}$ & $\%$ \\
\hline 1 & Name der Zeitung, Zeitschrift o.ä. & 112 & 49,34 & 131 & 43,23 & 243 & 45,85 \\
\hline 2 & Textsorte des Intertextes & 62 & 27,31 & 130 & 42,90 & 192 & 36,23 \\
\hline 3 & Medientyp des Intertextes & 36 & 15,86 & 30 & 9,90 & 66 & 12,45 \\
\hline 4 & Autor des Intertextes & 10 & 4,41 & 6 & 1,98 & 16 & 3,02 \\
\hline 5 & $\begin{array}{l}\text { Hinweis darauf, dass Zitat den Me- } \\
\text { dien entnommen ist }\end{array}$ & 7 & 3,08 & 6 & 1,98 & 13 & 2,45 \\
\hline Insgesamt & & 227 & 100 & 303 & 100 & 530 & 100 \\
\hline
\end{tabular}


Wie aus der Tabelle zu ersehen ist, werden am häufigsten Hinweise auf den Namen der Zeitung oder der Zeitschrift, der der Intertext entnommen ist, und auf die Textsorte des Intertextes gebraucht.

Die intertextuellen Fragmente, die zumindest einen von diesen metaintertextuellen Hinweisen enthalten, wurden für die weitere Analyse der pragmatischen Charakteristiken des massenmedialen Interdiskurses ausgewählt.

\section{Pragmalinguistische Charakteristiken der metaintertextuellen Markierungen des massenmedialen Interdiskurses in parlamentarischen Texten Deutschlands und Schwedens}

Neben den Hinweisen auf den Diskurstyp enthält der Metaintertext Informationen über die pragmatischen Intentionen des Redners. Wenn auch nicht in allen Fragmenten vorhanden, geben uns diese Informationen die Möglichkeit, bestimmte Schlussfolgerungen über die pragmatische Seite des Gebrauchs des massenmedialen Interdiskurses in den parlamentarischen Texten zu ziehen. Betrachten wir dazu folgendes Beispiel:

(2) Gerhard Schick (Bündnis 90 / Die Grünen): Wenn Sie es mir nicht glauben, dann vielleicht der Berichterstatterin der EVP-Fraktion - da sind die Christdemokraten im Europäischen Parlament versammelt -, Frau Wortmann-Kool - ich zitiere sie -: "Der Rat hat eine zu komplexe und fragile Prozedur für strauchelnde Banken erfunden. ... Mit so vielen beteiligten Leuten scheint es unmöglich, zielgerichtet zu handeln. ... Die Geschichte zeigt uns, dass Europa bei strauchelnden Banken schnell agieren muss." Recht hat sie. (DB: 1672)

Hier weist der Parlamentarier im Metaintertext explizit darauf hin, dass das Zitat aus den Massenmedien gebraucht wird, um die politischen Opponenten dazu zu bringen, dass sie an seine Worte glauben.

In diesem Zusammenhang lässt sich die Frage stellen, inwieweit das durch den Metaintertext explizit deklarierte Ziel des Gebrauchs des Intertextes dem wahren Ziel des Redners entspricht. Im vorherigen Beispiel kann man vermuten, dass das wahre Ziel des Redners ist, nicht vor den politischen Opponenten glaubwürdig zu erscheinen, sondern vor den Bürgern, die den Verlauf der Sitzung in den Massenmedien verfolgen.

Das Ziel, den zitierten Worten die Glaubwürdigkeit zu verleihen, kann durch den Hinweis auf die Unabhängigkeit und Autorität der Zeitung, die zitiert wird, erreicht werden.

(3) Dieter Janecek (Bündnis 90 / Die Grünen): „Bayern könnte zum Modell für eine gescheiterte Energiewende werden. " Das schreibt nicht die taz, sondern die FAZ, die linker Umtriebe unverdächtig ist. Sie sind auf dem besten Weg dazu - leider. (DB: 1037-1038) 
In diesem Beispiel wird dieses Ziel durch die Gegenüberstellung der zitierten Zeitung und einer anderen Zeitung erreicht. Der Redner unterstreicht, dass die Frankfurter Allgemeine Zeitung „linker Umtriebe unverdächtig ist“ und deshalb als eine objektive und zuverlässige Informationsquelle betrachtet werden kann.

Im Beispiel 4 weist die schwedische Politikerin darauf hin, dass das Zitieren eine gute Möglichkeit bietet, um „Debatte lebendiger zu machen”. Danach zitiert sie ihre politischen Opponenten:

(4) Anna Johansson (statsråd) (S): Låt mig läsa högt ur en artikel, för det kan ju vara ett spännande sätt att liva upp en interpellationsdebatt. "Citybanan är samtidigt det mest komplexa infrastrukturprojekt som någonsin planerats $i$ Sverige. Dess miljöpåverkan kan komma att bli betydande." Sedan drar man paralleller till Hallandsåsen. "Ett alternativ till Citybanan är att pröva om en ökad spårkapacitet kan påskyndas genom en ytspårslösning”" och så fortsätter man i samma stil. Alla var alltså inte överens om Citybanan. Denna artikel från 2006 är undertecknad av fyra personer som jag tror är bekanta för alla i kammaren: Fredrik Reinfeldt, Maud Olofsson, Lars Leijonborg och Göran Hägglund. (SR)

Auch hier kann man vermuten, dass das sprachlich deklarierte Ziel des Zitierens die richtigen kommunikativ-pragmatischen Intentionen des Politikers bestenfalls nur teilweise widerspiegelt.

Aus dem Beispiel 5 ist es deutlich zu sehen, dass die Intention, die der Redner explizit markiert, und das wahre Ziel des Gebrauchs des massenmedialen Interdiskurses unterschiedlich sind:

(5) Michael Grosse-Brömer (CDU/CSU): Ich empfehle Ihnen, ein bisschen von der Mitleidsnummer herunterzukommen. Dies sieht auch die ansonsten für sie wohlmeinende Presse so. Ich habe in der Süddeutschen Zeitung gelesen - ich zitiere -: Die Opposition "... setzt auf das Mitleid der Öffentlichkeit. ... Larmoyanz aber ist keine parlamentarische Tugend. ... In den Zeiten der Großen Koalition von 1966 bis 1969 war die Opposition noch viel kleiner, sie war nur halb so groß, sie bestand nur aus der FDP." [...] "Die zeigte aber damals, dass klein nicht mickrig bedeuten muss ... sie schaffte es deswegen, weil sie die Zeit nutzte, sich zu erneuern ..." Vielleicht hat die Süddeutsche Zeitung in dieser Hinsicht auch ein paar Anregungen für Sie. Ich möchte mich da gar nicht einmischen. (DB: 1020)

Hier macht der Politiker einen Ratschlag seinen politischen Gegnern. Es ist deutlich, dass sie diesem Ratschlag nicht folgen werden. Das richtige Ziel des Zitierens ist dagegen, den Eindruck auf die Zuschauer außerhalb des Plenarsaales zu machen, ihnen zu zeigen, dass die Opposition „larmoyant“ ist. Die explizite Markierung der pragmatischen Intention des Gebrauchs des Intertextes dient also oft eher dem Verhüllen der wahren Intentionen des Redners.

Pragmatisch bedingt ist der Hinweis der Parlamentsabgeordneten darauf, dass das Zitat in den Massenmedien den Vertretern ihrer politischen Opponenten gehört.

(6) Said Abdu (FP): Jag förstår de välfärdsföretag som nu hotas av regeringens politik. Men jag är inte ensam. Jag hade önskat att statsrådet hade lyssnat bättre till de egna leden. Det är 
nämligen så att den tidigare socialdemokratiska finansministern Kjell-Olof Feldt är tydlig $i$ sin kritik mot osäkerhetsregeringens utredningsdirektiv om vinster $i$ välfärden. I Dagens Nyheter den 4 april på s. 20 säger han: "Direktivet är formulerat så att det stänger utrymmet för normala företagsbeslut, till exempel om antalet anställda. Samtidigt finns en stor mängd företag inom vård, skola och omsorg som nu riskerar att bli utsatta för konfiskation. Det borde inte kunna ske i ett rättssamhälle, vilket jag hoppas att utredaren Ilmar Reepalu inser. " (SR)

Dadurch weist man darauf hin, dass die politischen Opponenten in einer bestimmten Frage nicht einig sind oder dass es eine Diskrepanz zwischen ihren Worten und Handlungen existiert.

Im deutschen Parlament werden Zitate gebraucht, die den politischen Gegnern gehören. Nach dem Zitieren wird es von dem Redner darauf hingewiesen:

(7) Nicole Maisch (Bündnis 90 / Die Grünen): Wir brauchen endlich ein Gesetz mit einer Obergrenze für Dispo-Zinsen. "Nie war Geld für die Banken so billig. Aber die Kunden ... müssen für ihren Dispo-Kredit im Durchschnitt über zehn Prozent zahlen. Das ist Abzocke!" [...] Es wundert mich, dass die SPD an dieser Stelle nicht klatscht; denn das waren nicht meine Worte, sondern die Worte ihres Parteivorsitzenden Sigmar Gabriel, die er der Bild-Zeitung zu Protokoll gegeben hat. (DB: 3092)

Dadurch wird gezeigt, dass die Abgeordneten im Deutschen Bundestag ihren Beifall nur dann ausdrücken, wenn der Redner zu ihrem politischen Lager gehört. Das Gesagte spielt dagegen eine untergeordnete Rolle.

Das Zitieren der Presse zeigt, dass der Politiker sich in der aktuellen politischen, wirtschaftlichen, sozialen Situation im Lande auskennt. Die Parlamentarier können den Opponenten vorwerfen, dass sie über die aktuellen Informationen nicht verfügen, wie es im Beispiel 8 zu sehen ist:

(8) Klaus Ernst (Die Linke): Die Frankfurter Rundschau beruft sich dabei auf geheime Dokumente des Wirtschaftsministeriums. Darin steht, so die FR: „Aus Sicht der Bundesregierung ist eine Übermittlung der endgültigen Textfassung " - das bezieht sich aufCETA - an die EU-Mitgliedstaaten „überfällig“. Wenn die Bundesregierung diesen Text hätte, dann bräuchte sie nicht um seine Übermittlung zu bitten. [...] Also, erzählen Sie doch nichts von Geheimhaltung, Herr Pfeiffer. Wollen Sie uns hier veräppeln? Lesen Sie doch wenigstens einmal die Presse! Dann wissen Sie, was in diesem Land los ist. (DB: 3026) Der Redner wirft seinem politischen Opponenten vor, dass er nicht weiß „was in diesem Lande los ist".

Der massenmediale Interdiskurs wird also von den deutschen und schwedischen Parlamentariern gebraucht, um den Aussagen die Glaubwürdigkeit zu verleihen und zu zeigen, dass man sich in der aktuellen Situation auskennt. Weiter wird der Versuch unternommen, die Pragmatik des Gebrauchs des massenmedialen Interdiskurses mithilfe der Analyse der Lexik mit der bewertenden Semantik im Metaintertext zu erklären. 


\section{Axiologische Charakreristiken der metaintertextuellen Markierungen des massenmedialen Interdiskurses in parlamentarischen Texten Deutschlands und Schwedens}

Neben der Analyse der expliziten Markierung der kommunikativ-pragmatischen Intentionen des Redners ermöglicht auch die nähere Betrachtung der Lexik mit der bewertenden Semantik den pragmatischen Wert des massenmedialen Interdiskurses in parlamentarischen Texten zu erklären.

Deswegen wurden aus den metaintertextuellen Markierungen des massenmedialen Interdiskurses Lexeme mit der bewertenden Semantik ausgesucht. Der Autor des Textes kann den Intertext und / oder seinen Autor positiv oder negativ bewerten, seinen Worten zustimmen oder sie widerlegen. Dabei hängt positive oder negative Bewertung sehr eng damit zusammen, welche soziale Rolle der Autor des Intertextes einnimmt. Positive Einstellung haben die Autoren des Textes zu den Autoren des Intertextes, wenn sie Journalisten, Wissenschaftler oder Vertreter der Bürgergesellschaft sind. Negativ werden dagegen in erster Linie politische Opponenten und ihre Worte bewertet.

Eine wichtige Rolle spielt dabei der Zusammenhang zwischen der bewertenden Bedeutung des Metaintertextes und des Intertextes. Betrachten wir dazu folgendes Beispiel aus dem Deutschen Bundestag:

(9) Andreas Schockenhoff (CDU/CSU): Sie sind, wie es die Frankfurter Allgemeine Zeitung richtig beschrieben hat, ,, das größte Bremsmanöver ..., seit das iranische Atomprogramm den Kinderschuhen entwachsen ist. " (DB: 144)

Hier enthält sowohl der Metaintertext, als auch der Intertext ein bewertendes semantisches Merkmal. Im Metaintertext ist das bewertende Lexem richtig zu finden. Mit seiner Hilfe wird positive Bewertung des Intertextes von dem Redner ausgedrückt. Andererseits ist der Intertext axiologisch gefärbt. Die negative Bewertung des Politikers aus der Opposition ist durch den Dysphemismus das größte Bremsmanöver ausgedrückt. Auf diese Weise unterstützt der Redner die Kritik gegen seine politischen Opponenten, die in der Zeitung angeführt ist.

Negative Einstellung hat man zu den Äußerungen der politischen Opponenten in den Medien. Diese Einstellung ist im Metaintertext explizit durch Lexeme mit bewertender Semantik ausgedrückt:

(10) Magnus Manhammar (S): Låt mig citera något Richard Jomshof skrev i SD-Kuriren 2009: "Tidningen rapporterar om en mörk framtid där den europeiska kontinenten hyser en allt större andel muslimer, vilket - om ingenting görs - oundvikligen kommer att leda till en omfattande islamisering och en framtid där det sekulära och demokratiska Europa står inför det värsta hotet någonsin - långt värre än tidigare hot från nazism och kommunism." Richard Jomshof menar, enligt citatet, att muslimer i Europa är ett värre hot än Hitlers utrotningsläger och Stalins Gulag. Det är ett fruktansvärt och hårresande citat. (SR) 
Dieses Beispiel enthält negative Bewertung des Zitats, die durch den Satz Det är ett fruktansvärt och hårresande citat (Das ist ein fürchterliches und haarsträubendes Zitat) ausgedrückt ist. Die negativen semantischen Merkmale enthalten die Lexeme ,fruktansvärd“" (,fürchterlich") und „,hårresande“ (,haarsträubend“). Der Autor des Zitates ist der politische Opponent.

Negative Bewertung kann durch den Ausdruck des Erstaunens wiedergegeben werden.

(11) Daniel Riazat (V): Vad säger då Sverigedemokraterna, partiet som står på det vanliga folkets sida? Jag kan citera från Svenska Dagbladet, där Stefan Jakobsson från Sverigedemokraterna säger: "Det skulle vara synd att ta bort skolverksamhet som fungerat väl förutom några få skandaler." Det är för mig väldigt märkligt att en politiker som arbetar med skolfrågor kan uttrycka sig på det sättet. (SR)

Hier wundert sich der schwedische Parlamentarier über den Ausdruck seiner politischen Opponenten. Dadurch schafft man den Eindruck, dass das Gesagte so schlecht ist, dass man daran nicht glauben kann.

Die Parlamentsabgeordneten können die Worte ihrer politischen Opponenten positiv bewerten, um auf den Widerspruch zwischen dem Gesagten und ihren Handlungen hinzuweisen:

(12) Diana Golze (Die Linke): Auch wenn die Ministerin selber nicht dazu Stellung nehmen kann, will ich für alle Anwesenden ein Zitat aus einer Pressemitteilung vom 10. September dieses Jahres anführen, in der sie erklärt hat: „Das Betreuungsgeld ist grundsätzlich falsch und richtet in seiner fehlerhaften Ausgestaltung viel Schaden an. "[...] Diese Auffassung teile ich uneingeschränkt. (DB: 312)

In diesem Beispiel teilt der Abgeordnete aus der Opposition die Auffassung der Ministerin.

Wie es hier zu sehen ist, trägt die Analyse der bewertenden semantischen Merkmale, die im Metaintertext vorhanden sind, zum Verständnis der pragmatischen Intentionen der Redner bei. Abschließend wird der Gebrauch der metaintertextuellen Markierungen in parlamentarischen Texten Deutschlands und Schwedens verglichen.

\section{Vergleichende Analyse der metaintertextuellen Markie- rungen des massenmedialen Interdiskurses in parlamentari- schen Texten Deutschlands und Schwedens}

In diesem Paragraphen wird versucht, festzustellen, welche Gemeinsamkeiten und Besonderheiten die metaintertextuellen Markierungen des massenmedialen Interdiskurses in parlamentarischen Texten Deutschlands und Schwedens aufweisen.

Die größte Gruppe der metaintertextuellen Markierungen des massenmedialen Diskurses bilden die Massenmediennamen (s. Tab. 1). Für diese Gruppe wur- 
de eine Reihe von Kriterien für den Vergleich ausgewählt. ${ }^{2} \mathrm{Zu}$ diesen Kriterien gehören folgende:

1. Örtliche Zuordnung der Massenmedien:

a) Einheimisches Massenmedium - ausländisches Massenmedium;

b) Für die einheimischen Massenmedien: Überregionales Massenmedium regionales Massenmedium - lokales Massenmedium.

2. Thematische Zuordnung der Massenmedien: Massenmedium der allgemeinen Thematik - Zeitung / Zeitschrift mit der fachlichen Thematik.

3. Zuordnung der Massenmedien nach dem Medientyp:

a) Zeitung - Magazin - Rundfunk - Fernsehen.

b) Gedruckte Ausgabe - Online-Ausgabe.

Die Analyse nach diesen Kriterien ermöglicht es, folgende Gemeinsamkeiten des Gebrauchs der metaintertextuellen Hinweise im deutschen und schwedischen Parlament festzustellen:

1. In beiden Parlamenten werden am häufigsten Zeitungen und Zeitschriften zitiert (96,43\% im deutschen Parlament und 94,66\% im schwedischen Parlament). Relativ selten werden Zitate aus Rundfunk- und Fernsehsendungen (3,57\% respektive 5,34\%) gebraucht.

Zu den im schwedischen Parlament am häufigsten zitierten Zeitungen gehören folgende: „Dagens Nyheter“, „Svenska Dagbladet“, „Dagens Industri“, „Aftonbladet“, „Dagens Samhälle“, „Expressen“ und „Göteborgs-Posten“. Das sind die populärsten Tages- und Abendzeitungen in Schweden (vgl. Svenska mediehus 2014/2015).

In den parlamentarischen Texten Deutschlands werden am häufigsten folgende Zeitungen und Zeitschriften zitiert: „Frankfurter Allgemeine Zeitung“, „Der Spiegel“, „Süddeutsche Zeitung“, „Handelsblatt", „Das Bild“, „taz“, „Die Zeit“. Auch sie gehören zu den am meisten gelesenen Zeitungen und Zeitschriften in Deutschland (s. z.B. Statista).

2. In beiden Parlamenten werden vorwiegend einheimische Medien zitiert (95,36\% im deutschen Parlament und 99,24\% im schwedischen Parlament). Zitate aus ausländischen Massenmedien (4,64\% respektive 0,76\%) werden meistens bei der Besprechung der außenpolitischen Themen gebraucht. Solche Zitate können in der Originalsprache wiedergegeben werden.

3. $\mathrm{Zu}$ den Textsorten, die am häufigsten in den beiden Parlamenten zitiert werden, gehören Artikel, Interview und Pressemitteilung. Häufig werden Überschriften zitiert. Zu den häufigsten Hinweisen, die die Textsorte markieren, zu der der Intertext gehört, gehören im schwedischen Parlament artikel und seine Abart debattartikel, intervju, pressmeddelande und rubrik. Es sind auch allgemeine Hinweise darauf zu finden, dass der Intertext in den Medien oder in der Zeitung veröffentlicht wurde: massmedier, medier, tidning.

${ }^{2}$ Vgl. Richter (1989: 72-76) 
4. In den parlamentarischen Texten Deutschlands und Schwedens sind fast keine Zitate aus den parlamentarischen Zeitungen zu finden.

Das Vergleichen von dem Metaintertext erlaubt uns, folgende Unterschiede zwischen seinem Gebrauch im deutschen und im schwedischen Parlament festzustellen:

1. Im schwedischen Parlament werden am häufigsten Zitate aus nur zwei größten Tageszeitungen gebraucht: „Dagens Nyheter” und „Svenska Dagbladet”. Es gibt dagegen mehrere Zeitungen und Zeitschriften, die im deutschen Parlament häufig zitiert werden: „Frankfurter Allgemeine Zeitung”, „Der Spiegel”, „Süddeutsche Zeitung”, „Die Zeit”, „Die Welt“.

2. Im deutschen Parlament kommen häufiger Hinweise darauf vor, dass die Online-Ausgabe des Massenmediums zitiert wird. Sie sind folgenderweise markiert:

a) Durch den Hinweis darauf, dass es eine Online-Ausgabe ist.

b) Durch den Gebrauch von der Webadresse.

3. Im schwedischen Parlament sind mehrere Zitate aus den spezialisierten fachlichen Zeitungen und Zeitschriften zu finden. Thematik der Medien entspricht den Themen, die im Plenum verhandelt werden, z. B. „Driva Eget“ bei der Behandlung von wirtschaftlichen Themen oder „Gotlands Vindelsproducenters medlemsnytt" bei der Diskussion zu erneuerbaren Energiequellen. Im Bundestag werden dagegen vorwiegend Zitate aus den überregionalen und regionalen Medien der allgemeinen thematischen Ausrichtung gebraucht.

\section{Fazit und Ausblick}

Zusammenfassend ist festzuhalten, dass Metaintertext zahlreiche Informationen sowohl über den Intertext selbst, als auch über die pragmatischen Intentionen des Redners, der den Intertext gebraucht, liefert. Zu diesen Informationen gehört der Hinweis auf den Diskurstyp des Intertextes, was die Verwendung des Metaintertextes bei der Erforschung der Interdiskursivität ermöglicht.

Die Untersuchung der metaintertextuellen Markierungen des massenmedialen Interdiskurses zeugt von seiner wichtigen Rolle sowohl in der Parlamentskommunikation Deutschlands, als auch in der Parlamentskommunikation Schwedens. Der Gebrauch des massenmedialen Inderdiskurses in parlamentarischen Texten ist von seinem pragmatischen Potenzial bedingt. Der massenmediale Interdiskurs hilft dem Redner, seine kommunikativ-pragmatischen Ziele zu erreichen: seine eigene Kompetenz in den aktuellen Fragen zu zeigen und bei der Kritik der politischen Opponenten glaubwürdig zu erscheinen. Dabei können die Intentionen des Redners durch Metaintertext sprachlich explizit markiert werden. Neben der Analyse dieser Markierungen trägt auch die nähere Betrachtung der bewertenden 
Semantik des Metaintertextes zum Verständnis der pragmatischen Intentionen der Redner bei.

In der Parlamentskommunikation Deutschlands gebrauchen die Abgeordneten den massenmedialen Interdiskurs vorwiegend, um die politischen Gegner zu kritisieren. Die Kritik erfolgt durch die Auswahl der Zitate aus den größten überregionalen Zeitungen und Zeitschriften. In der Parlamentskommunikation Schwedens versuchen die Abgeordneten dagegen in erster Linie, sich selbst im positiven Licht zu präsentieren. Das geschieht u.a. durch den Gebrauch von Zitaten aus spezialisierten Zeitungen und Zeitschriften. Auf diese Weise wird gezeigt, dass der Parlamentsabgeordnete sich in dem jeweiligen Thema gut auskennt, dass er in der debattierten Frage kompetent ist und dass man demzufolge seinen Worten glauben kann.

Bei diesem Thema ergibt sich das Potenzial für zukünftige Studien, die z.B. der diachronischen Untersuchung der Interdiskursivität in der parlamentarischen Kommunikation gewidmet werden können. Eine solche Untersuchung soll ermöglichen, die Entwicklungsprozesse im Bereich der parlamentarischen Kommunikation besser zu verstehen. Dabei kann sowohl expliziter Intertext mit dem Metaintertext, als auch impliziter Intertext analysiert werden.

\section{Literatur}

Allen, Graham: Intertextuality. London, New York 2000.

Bachtin, Michail: Проблема содержания материала и формы в словесном художественном творчестве. In: Вопросы литературы и эстетики. Москва 1975. S. 6-71.

Burkhardt, Armin: Das Parlament und seine Sprache: Studien zu Theorie und Geschichte parlamentarischer Kommunikation. Tübingen 2003.

Burkhardt, Armin: Zwischen Diskussions- und Schaufensterparlamentarismus. Zur Diagnose und Kritik parlamentarischer Kommunikation - am Beispiel von Zwischenfragen und Kurzdialogen. In: Dörner, Andreas / Vogt, Ludgera (Hrsg.): Sprache des Parlaments und Semiotik der Demokratie: Studien zur politischen Kommunikation in der Moderne. Berlin, New York 1995. S. 73-107.

DB = Deutscher Bundestag: Stenografischer Bericht. 18. Legislaturperiode. Sitzung 1-42. Berlin. http://dip21.bundestag.de [Zugriff am 5.08.2015].

Fairclough, Norman: Discourse and social change. Cambridge 1992.

Gavenko, Agnessa: Метаинтертекстуальность в художественном тексте (на материале русских рассказов 80-х годов XX-XXI веков). In: Филология и человек 2, 2011, S. 36-48.

Heinemann, Wolfgang: Zur Eingrenzung des Intertextualitätsbegriffs aus textlinguistischer Sicht. In: Klein, Josef / Fix, Ulla (Hrsg.): Linguistische und literaturwissenschaftliche Beiträge zur Intertextualität. Tübingen 1997. S. 21-37.

Jarren, Otfried / Donges, Patrick: Politische Kommunikation in der Mediengesellschaft. Wiesbaden 2006.

Kristeva, Julia: Desire in Language: A Semiotic Approach to Literature and Art. New York 1980. 
Oliveira, Sara: The Unthinkable Unprecedented: Intertextuality in Newpapers Genres. In: Linguagem em (Dis)curso 1 (5), 2004, S. 9-28.

Richter, Hermann: Pressekonzentration und neue Medien: der Einfluss neuer Wettbewerbsimpulse auf die Konzentration bei Tageszeitungen. Göttingen 1989.

Schewtschenko, Iryna: Інтердискурсивність політичного дискурсу. In: Вісник ХНУ 848, 2009, S. 53-57.

$\mathrm{SR}=$ Sveriges Riksdag: Riksdagens protokoll. 2013/14, 2014/15. http://www.riksdagen.se/Webbnav/ index.aspx?nid=101\&bet=2008/09:36 [Zugriff am 19.08.2015].

Statista. Verkaufte Auflage der überregionalen Tageszeitungen in Deutschland im 2. Quartal 2015. http:/de.statista.com/statistik/daten/studie/73448/umfrage/auflage-der-ueberregionalen-tageszeitungen/ [Zugriff am 23.08.2015].

Strömbäck, Jesper: Makt, medier och samhälle: en introduktion till politisk kommunikation. Stockholm 2009.

Svenska mediehus 2014/2015: Fakta om marknad och medier. Stockholm 2015.

Wu, Jianguo: Understanding Interdiscoursivity: A Pragmatic Model. In: Journal of Cambridge Studies 2-3 (6), 2011. S. 95-115.

\section{Abstracts}

Im vorliegenden Beitrag werden die semantischen und pragmatischen Charakteristika der metaintertextuellen Markierungen des medialen Interdiskurses in den parlamentarischen Texten Deutschlands und Schwedens untersucht. Interdiskursive Zitate werden von dem Metaintertext begleitet, d.h. von Textfragmenten, die den Intertext beschreiben. Zitate in den parlamentarischen Texten, die den massenmedialen Diskurs repräsentieren, wurden mithilfe der semantischen Analyse des Metaintertextes ausgesondert. Es wurde gezeigt, auf welche Weise der Metaintertext für die Analyse der pragmalinguistischen Besonderheiten des Gebrauchs des Interdiskurses verwendet werden kann. Anschließend wurde der Gebrauch von metaintertextuellen Markierungen des massenmedialen Interdiskurses in den parlamentarischen Texten Deutschlands und Schwedens verglichen.

Schlüsselwörter: Interdiskursivität, massenmedialer Diskurs, Metaintertext, parlamentarischer Text

\section{Metaintertextual markers of the mass media interdiscourse in the parliamentary texts of Germany and Sweden}

The article deals with the semantic and pragmatic characteristics of metaintertextual markers of mass media interdiscourse in the parliamentary texts of Germany and Sweden. Interdiscoursive citations are accompanied by metaintertext, e.g. the fragments of text that describe intertext. Citations in the parliamentary texts that represent mass media discourse are selected through the semantic analysis of metaintertext. Application of metaintertext to the analysis of pragmatic characteristics of interdiscourse usage is demonstrated. The usage of the metaintertextual markers of mass media interdiscourse in the parliamentary texts of Germany and Sweden is compared.

Keywords: interdiscoursivity, mass media discourse, metaintertext, parliamentary text 
Oleksandr Stasiuk

Nationale Taras-Schewtschenko-Universität Kyjiw

Institut für Philologie

bulw. Tarasa Schewtschenka 14

01601 Kyjiw

Ukraine

E-Mail: oleksandr-stasiuk@ukr.net 